\title{
Short-term and long-term habituation of the acoustic startle response as a function of stimulus rise time in rats
}

\author{
PETER K. D. PILZ \\ University of Tübingen, Tübingen, Germany \\ and \\ ROBERT N. LEATON \\ Dartmouth College, Hanover, New Hampshire
}

\begin{abstract}
In three experiments, short-term habituation (STH) and long-term habituation (LTH) of the acoustic startle response (ASR) were assessed as a function of the rise time of the startle-inducing stimulus. In Experiment 1, presentations of a 300-msec rise time stimulus (RTS) produced neither a startle response nor response decrement to a 0.4-msec RTS when switched to that stimulus within a session (STH). When tested over days (LTH) training with the 300 -msec RTS produced significant response decrements to the 0.4 -msec RTS. Stimulus-induced freezing and vocalization were inversely related to rise time, and these emotional responses habituated over days. Experiment 2 showed that STH was inversely related to the rise time of the training stimulus, not simply to stimulus change. Experiment 3 replicated the LTH results of Experiment 1. These data show that STH of ASR is inversely related to stimulus rise time, whereas LTH is relatively independent of rise time.
\end{abstract}

The acoustic startle response (ASR) is a short-latency response elicited by a sudden loud acoustic stimulus. In the rat, the latency for the response, measured in the hindlimb muscles, is $8-10 \mathrm{msec}$, suggesting a relatively simple underlying neural circuitry (Davis, Gendelman, Tischler, \& Gendelman, 1982). Although there is some disagreement in detail, all the proposals for the neural circuits for the ASR require only a few central synapses and assume that the caudal pontine reticular nucleus (PnC) or a closely associated pontine nucleus represents the motor output from the brain. The circuits include the auditory nerve, the ventral cochlear nucleus and/or cochlear root neurons, one or two synapses in the brainstem, a spinal interneuron, a motoneuron, and a muscle (Kandler \& Herbert, 1991; Lee, Lopez, Meloni, \& Davis, 1996; Lingenhöhl \& Friauf, 1994; Yeomans \& Frankland, 1996).

Despite the apparent simplicity of this circuitry, the ASR shows various forms of plasticity, including short-

Portions of this research were presented at the 26th Annual Meeting, Society for Neuroscience, Washington, D. C., November, 1996. This research was supported by the Deutsche Forschungsgemeinschaft (DFG: SFB 307 C2) and by Dartmouth College. Experiments 1 and 2 were conducted while R.N.L. was visiting professor of animal physiology, Faculty for Biology, University of Tübingen, and thanks are due Professor H.-U. Schnitzler for his generous support. We thank Allison Ahart for testing the animals in Experiment 3. Correspondence concerning this article should be addressed to P. Pilz, Tierphysiologie, Universität Tübingen, Morgenstelle 28, D-72076 Tübingen, Germany, or to R. N. Leaton, Department of Psychology, Dartmouth College, Hanover, NH 03755 (e-mail: peter.pilz@uni-tuebingen.de or leaton@dartmouth.edu). term habituation (STH) and long-term habituation (LTH; Davis \& File, 1984). STH is defined as response deficits that occur within a test session. These deficits are inversely related to the length of the interstimulus interval (ISI) and show complete spontaneous recovery over a few minutes (Davis \& File, 1984; Thompson \& Spencer, 1966). It is generally assumed that STH occurs as some form of synaptic depression within the basic startle circuit. The data supporting such an intrinsic process are indirect but are generally consistent with that view (Davis \& File, 1984; Pilz \& Schnitzler, 1996).

LTH is defined as relative permanent response decrements that are detected between test sessions and may last for days, weeks, or months (Leaton, 1976). Both behavioral (Leaton, 1976) and neurophysiological (Jordan, 1989; Leaton \& Supple, 1986) data indicate that LTH and STH are independent processes that require independent neural mechanisms. Brain lesions that block LTH of ASR do so without affecting the basic startle response or the STH of the response. Such data have led to the assumption that LTH is an extrinsic process, involving neural pathways outside the basic startle circuitry that induce some form of inhibition on that circuitry (see, e.g., Jordan, 1989; Leaton \& Supple, 1986).

The threshold for elicitation of the ASR and the amplitude of the response depend critically on the intensity and rise time of the acoustic stimulus (Pilz, 1989; Pilz, Schnitzler, \& Menne, 1987). (The specific critical variable is the energy of the acoustic stimulus integrated over the first 10 msec or so of stimulus duration [Fleshler, 1965; Marsh, Hoffman, \& Stitt, 1973], but for stimuli longer 
than about $10 \mathrm{msec}$, this variable is completely confounded with the intensity and rise time of the acoustic stimulus. We will refer to intensity and rise time for convenience.) Giant neurons in the PnC mirror many of the behavioral characteristics of the ASR in both the rat (Lingenhöhl \& Friauf, 1994) and the cat (Wu, Suzuki, \& Siegel, 1988). These neurons have high firing thresholds to acoustic stimuli, and their thresholds are very sensitive to stimulus rise time. It is just these giant PnC neurons that appear to be a critical part of the ASR circuitry (Koch, Lingenhöhl, \& Pilz, 1992).

If STH of the ASR is intrinsic to the basic startle circuitry, it should depend on the degree of activation of the neurons in that circuitry, either at the PnC or afferent to the PnC. Thus, STH should be sensitive to the rise time of the acoustic stimulus. Since LTH, unlike STH, appears to be an extrinsic process, it is less obvious that it should depend critically on stimulus rise time. LTH might depend on initial activation of the same neural circuitry as STH prior to activation of the extrinsic circuitry, and it, too, would then vary significantly with stimulus rise time. On the other hand, the acoustic stimulus might reach the extrinsic circuitry through independent neural processes with different parametric characteristics. The present experiments were designed to study the relationship between the rise time of the acoustic stimulus and both short-term and long-term habituation of the ASR.

\section{EXPERIMENT 1}

In a preliminary experiment, Pilz (1989) tested startle responsiveness to an acoustic stimulus with a $0.4-\mathrm{msec}$ rise time following training with either a $0.4-\mathrm{msec}$ rise time stimulus (RTS) or a 72-msec RTS. Rats were significantly less responsive following training with the 0.4msec RTS than following training with the $72-\mathrm{msec}$ RTS $\mathrm{STH}$, indexed by responding during the test series, was inversely related to stimulus rise time. This is the expected relation for an intrinsic STH process that should be directly related to the degree of excitation within the startle circuit. Experiment 1 was designed to extend this finding to better controlled test conditions for STH and to examine the relation of stimulus rise time to LTH. STH was assessed with between-group comparisons following stimulus rise time changes within a session. LTH was assessed following stimulus rise time changes between sessions. For both comparisons, a stimulus was used for the slow rise condition that was expected to produce little or no startle response and, thus, little or no STH.

\section{Method}

Subjects. Thirty-six male albino rats (Wistar) were kept in groups of 4 animals per cage and maintained on a 12:12-h light: dark cycle, lights on at 7:00. They weighed between 200 and $300 \mathrm{~g}$ and had free access to food and water in their home cages. Testing took place between 12:30 and 17:00.

Apparatus. Four startle chambers were used, allowing the simultaneous testing of 4 animals. The apparatus was similar to that described by Pilz and Schnitzler (1996). The rats were placed in wire mesh cages $(8 \times 9 \times 10 \mathrm{~cm})$, which were mounted on piezoelectric accelerometers. The voltage output of the accelerometer was amplified and digitized and then analyzed by a microcomputer system. The cages were housed within separate sound-attenuating chambers $(70 \times 50 \times 37 \mathrm{~cm})$, which were illuminated by a cold light bulb ( $5 \mathrm{~W})$. Acoustic stimuli were computer generated with a function synthesizer (Hortman, UFS), amplified, and delivered through a piezohorn speaker (Motorola, KSN 1025) mounted $38 \mathrm{~cm}$ from the test cage. Acoustic intensities were measured with a $1 / 2$-in. condenser microphone and a measuring amplifier (Brüel and Kjaer). One of the chambers was equipped with a video camera (Conrad Electronics), to allow continuous observation of the animal from a remote monitor, and with the $1 / 2$-in. condenser microphone, to allow recording of the rat's ultrasonic vocalizations (Kaltwasser, 1990 ). The vocalizations, which were in the $22-\mathrm{kHz}$ range, were converted to the audible frequency range with a voltage-controlled amplifier (Wavetek, model 136) and monitored with remote earphones.

The stimuli were $10-\mathrm{kHz}, 105-\mathrm{dB}$ SPL (RMS) tone bursts. The slow-rise stimulus had a $300-\mathrm{msec}$ rise/fall time. The fast-rise stimulus had a $0.4-\mathrm{msec}$ rise/fall time. Stimulus duration was $100 \mathrm{msec}$ plus the rise/fall time: $100.8 \mathrm{msec}$ for the fast-rise stimulus and $700 \mathrm{msec}$ for the slow-rise stimulus. The rise/fall times were linear. Startle amplitude was taken to be the difference between the maximum voltage for a fixed time window before and after stimulus onset. Two time windows were recorded on each trial, 80 and $380 \mathrm{msec}$ (i.e., $80 \mathrm{msec}$ plus the slow-rise time). The stimuli were superimposed on a $45-\mathrm{dB}$ SPL background white noise (bandwidth $2-30 \mathrm{kHz}$, variation $\pm 5 \mathrm{~dB}$ ) delivered by a second speaker (Matsushita, model EAS TH400).

Procedure. The rats were divided into three groups of 12 , matched for body weight, and each rat was handled for 1 min each day for 3 days. They were then given three daily 5-min preexposure sessions in the startle chambers, with no stimulation other than the background white noise. On Day 1 , Group 1 received 40 presentations of the 0.4-msec RTS, and both Group 2 and Group 3 received 40 presentations of the $300-m s e c$ RTS. The stimuli on this and all subsequent days were presented on a $20-\mathrm{sec}$ ISI, and the first stimulus of a session was presented 2 min into the session. On Day 2, Group 1 again received 40 presentations of the $0.4-\mathrm{msec}$ RTS. Group 2 received presentations of the 300-msec RTS for Trials $1-20$, and then the stimulus was changed to the $0.4-\mathrm{msec}$ RTS for Trials $21-40$. Group 3 again received 40 presentations of the 300 msec RTS. Day 2 allowed for the comparison of STH after 20 trials with a 0.4-msec RTS (Group 1) or 20 trials with a 300-msec RTS (Group 2) and for the possibility of additional LTH in Group 3. Only 20 trials were run on Day 3, and only Groups 1 and 3 were tested. Group 1 continued to receive the 0.4 -msec RTS. Group 3 received presentations of the 0.4-msec RTS for the first time and on all the trials to assess the possibility that LTH had developed during trials with the $300-\mathrm{msec}$ RTS on Days 1 and 2. All the treatments were given on consecutive days.

During each experimental session, freezing and vocalizations were measured in 1 of the 4 simultaneously tested rats. Freezing was defined as the absence of all movements of the rat, including vibrissae, except those movements that were due to respiration or vocalization (Fanselow, 1980). The beginning and end of each bout of freezing was manually indicated on a computer that recorded total duration of freezing. Rats vocalize with discrete calls (Kaltwasser, 1990), and each discrete episode of vocalization was manually indicated, allowing the recording of the total number of vocalizations during a session.

Trial sequences were statistically analyzed, using a mixed between-within repeated measures analysis of variance (ANOVA). Linear (first-order) polynomial contrasts were used to assess trial effects and interactions. Usually, only the first few trials of a sequence were analyzed, because continuing asymptotic performance diluted the comparisons. For the analysis of single-trial startle data, 


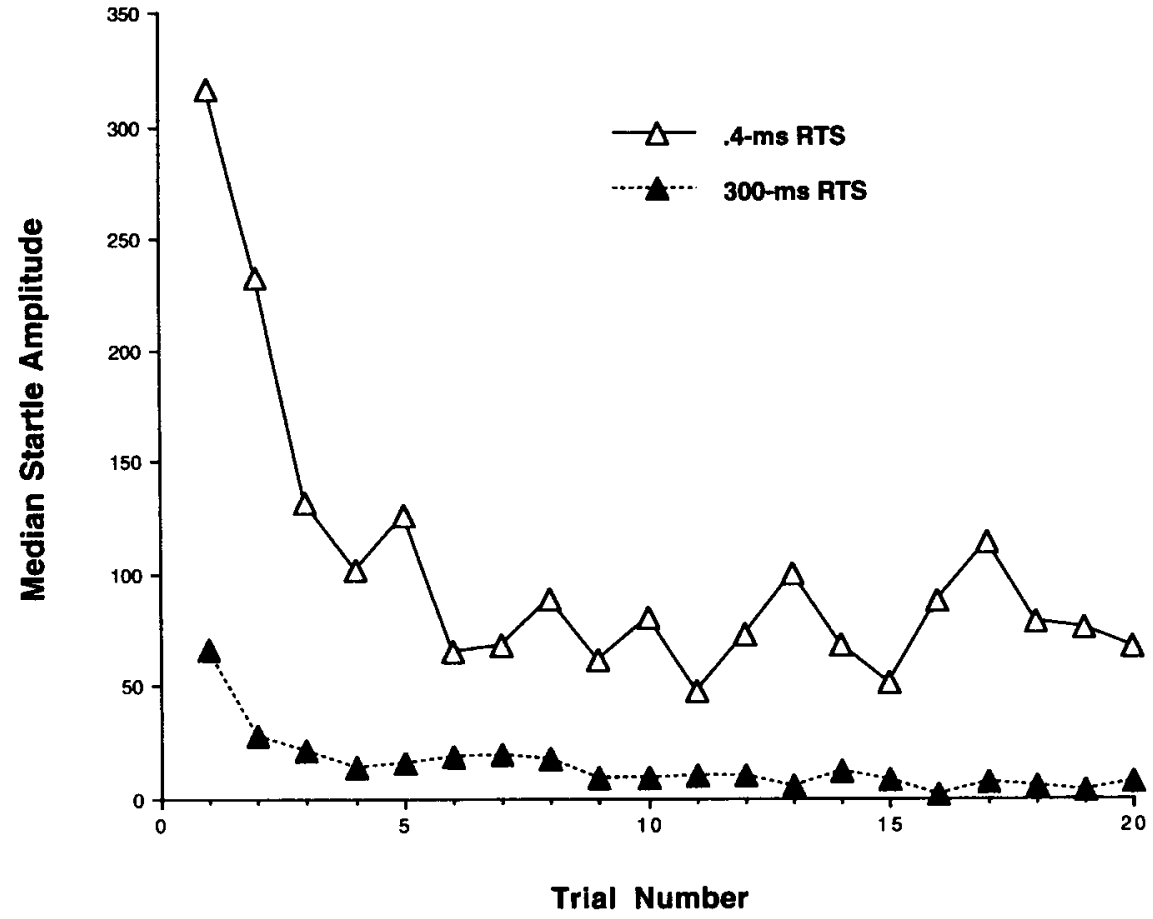

Figure 1. Median startle amplitude in the 380 -msec poststimulus response window to a 0.4-msec rise time stimulus (RTS; $n=12)$ or a 300-msec RTS $(n=24)$ on Day 1 in Experiment 1. (The startle data are displayed as medians throughout Experiment 1 because this measure more clearly reflected the statistical analyses.)

a square-root transform was applied to help stabilize the variances, because of the inherent variability of such single-trial startle data. Independent and dependent $t$ tests were evaluated with a two-sided probability.

\section{Results and Discussion}

Difference in acoustic startle response amplitude to fast and slow rise time stimuli. Figure 1 shows the difference in startle amplitude to the 0.4-msec RTS and the 300 -msec RTS for the first 20 trials on Day 1 for the 380 msec response window. (Only the first 20 trials are shown, because little or no change occurred after that time. For the purposes of this comparison, Groups 2 and 3 were combined, since they were treated identically on Day 1.) As was expected, the 0.4-msec RTS produced significantly greater response than did the 300 -msec RTS. Analysis over the first 10 trials showed a significant difference between groups $[F(1,34)=74.32, p<.001]$, a significant decrease over trials $[F(1,34)=48.21, p<.001]$, and a significant group $\times$ trial interaction $[F(1,34)=$ $29.36, p<.001]$. The $300-\mathrm{msec}$ RTS did produce a significant level of response over the first few trials, and analysis of that group alone yielded a significant trial effect $[F(1,23)=32.63, p<.001]$.

The rather long $380-\mathrm{msec}$ poststimulus-onset response window did not affect responsiveness measured to the $0.4-\mathrm{msec}$ RTS. The response to the 0.4-msec RTS in this long response window did not differ significantly from the response in the 80-msec window $(F<1)$. The 300 msec RTS produced no detectable response in the 80 msec response window. For all further analyses, only the data from the $380-\mathrm{msec}$ response window will be reported.

Short-term habituation following training with either a 0.4-msec or a 300-msec rise time stimulus. Figure 2 shows the Day 2 test results for Groups 1 and 2 . (Group 3 is not shown. The animals in that group continued to receive the 300 -msec RTS for all 40 trials and showed very low response amplitudes throughout.) The best indication of any difference in STH following training with the $0.4-\mathrm{msec}$ or $300-\mathrm{msec}$ RTS is the response of the two groups on the first few trials after the rise time change. Beginning on Trial 21, both groups received the same 0.4-msec RTS. Group 2 responded significantly more than Group 1 on Trial 21 [ $t(22)=2.54, p<.02]$ and over the two $[F(1,22)=5.73]$, three $[F(1,22)=4.52]$, and four $[F(1,22)=4.49]$ trial blocks beginning with Trial 21 (all $p s<.05$ ). The groups showed comparable response amplitudes over the last 10 or 15 trials, since the common rise time stimulation eliminated group differences.

The degree of STH produced by training with the 300 msec RTS can be shown by comparing the first $0.4-\mathrm{msec}$ RTS trials for Group 2 (beginning with Trial 21) with the initial trials of the session for Group 1 (beginning with Trial 1). The Trial 1 versus Trial 21 comparison was not significant $[t(22)=1.12, p>.25]$, nor did any of the trialblock comparisons described above yield significant 


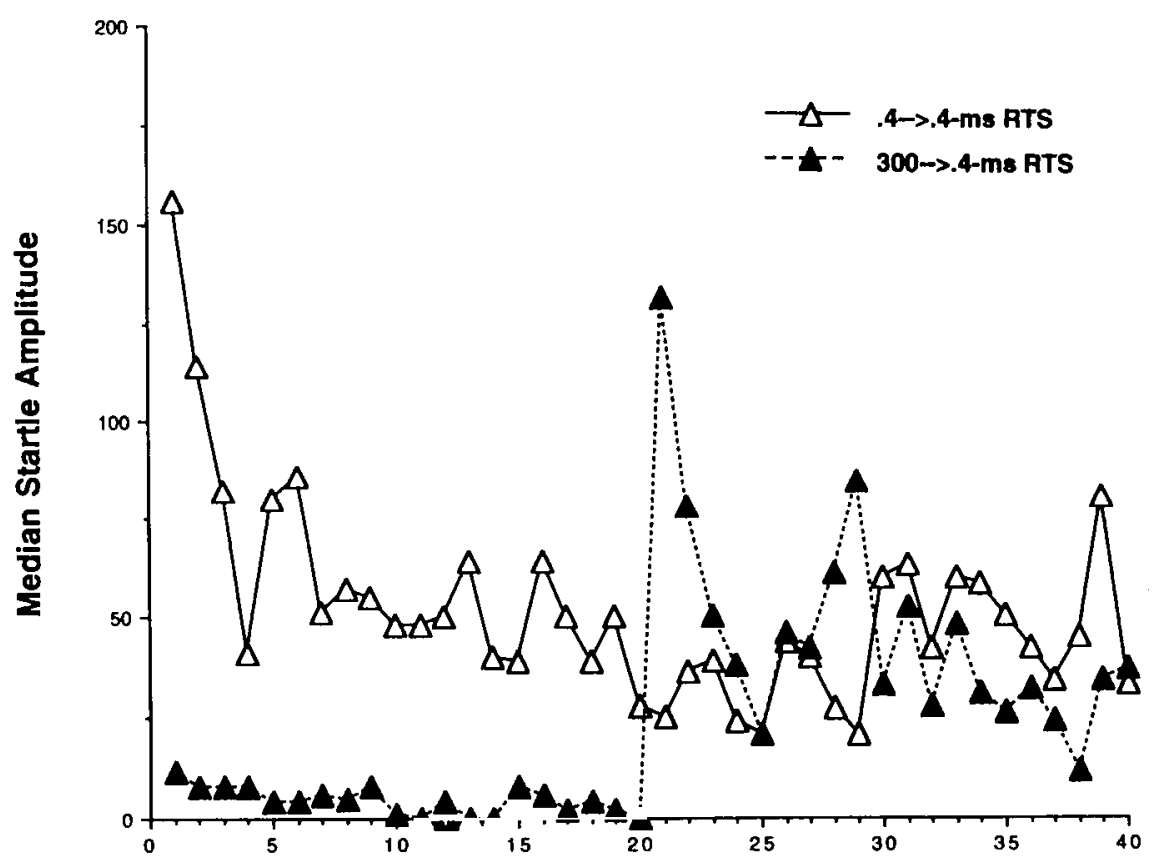

Trial Number

Figure 2. Median startle amplitude on Day 2 in Experiment 1. The 0.4-msec rise time stimulus (RTS) group (Group $1, n=12$ ) received a 0.4-msec RTS on all trials. The 300-msec RTS group (Group 2, $n=12$ ) received a 300-msec RTS on Trials 1-20 and a 0.4-msec RTS on Trials $21-40$.

group differences or interactions [largest $F(1,22)=1.51$, $p>.20]$. The lack of significant differences in these comparisons suggests that the $300-\mathrm{msec}$ RTS produced no STH over the initial 20 trials of the session.

These data confirm the preliminary data (Pilz, 1989) showing that STH is inversely related to the rise time of the startle-inducing stimulus. Training with the $300-\mathrm{msec}$ RTS, a stimulus that produced little or no response, produced no detectable STH. This is just the relationship one would expect if the mechanism for STH lies within the startle circuit and is directly related to the degree of activation of that circuit.

Long-term habituation following training with either a 0.4-msec or a 300-msec rise time stimulus. The first indication that the different rise time stimuli produced different effects on STH and LTH was seen in Group 2 on Day 2. Although training with the 300-msec RTS produced no detectable STH in Group 2, this long RTS did appear to produce LTH. The responsiveness of Group 2 to the first presentation of the 0.4-msec RTS on Day 2, Trial 21 (Figure 2) was significantly lower than the responsiveness of Group 1 to that stimulus on Trial 1, Day 1 [Figure $1 ; t(22)=2.32, p<.05]$.

LTH was assessed in Group 3 with the change in rise times occurring between rather than within sessions. Figure 3 shows the Day 3 test results for Groups 1 and 3, with the Day 1 data for Group 1 shown for comparison.
On Day 3 both Group 1 and Group 3 were tested under identical conditions with the $0.4-\mathrm{msec}$ RTS. Group 1 had received 2 previous days of training ( 80 trials) with the 0.4-msec RTS, and Group 3 had received 2 previous days of training with the 300 -msec RTS ( 80 trials). Comparisons between Group 1 and Group 3 on Day 3 should reveal any differences in LTH produced by the different training conditions, whereas comparisons between Day 3 and Day 1 should reveal the extent of LTH produced by these test conditions.

As can be seen in Figure 3, Groups 1 and 3 showed very similar response levels over all trials on Day 3. Neither the first trial difference $(t<1)$ nor the difference over Trials $1-10[F(1,22)=2.12, p>.15]$ was significant. Comparisons over Trial Blocks 2, 3, 4, or 5 did not approach significance for either the group difference or the interaction [largest $F(1,22)=1.03, p>.30$ ].

In comparing Day 1 and Day 3 for these two groups, only the first trial comparisons failed to reach significance. Comparisons over Trials 1 and 2 on Day 1 and Day 3 were significant for both groups [Group $1, F(1,11)=$ $29.61, p<.001$; Group 3, $F(1,22)=4.53, p<.05]$. In addition, comparisons of either group to Group 1, Day 1 over Trial Blocks 3, 4, 5 or 10 yielded significant group differences [for Group 1, smallest $F(1,11)=35.69, p<$ .001 ; for Group 3, smallest $F(1,22)=7.35, p<.05]$. However, the first trial differences between Days 1 and 3 did 


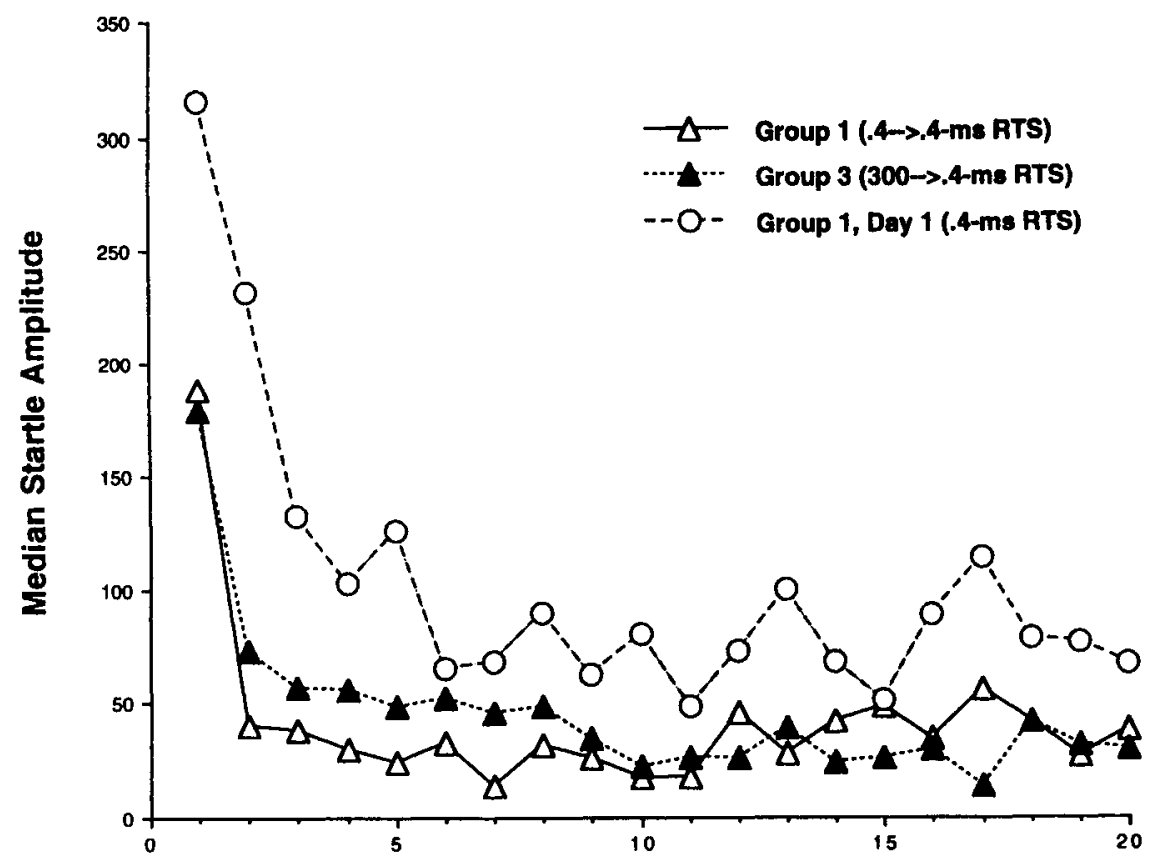

Trial Number

Figure 3. Median startle amplitude to a 0.4-msec rise time stimulus (RTS) on Day 3 in Experiment 1. On each of Days 1 and 2, Group $1(n=12)$ had received 40 trials with a 0.4-msec RTS, and Group $3(n=12)$ had received 40 trials with a 300-msec RTS. Responsiveness of Group 1 to a 0.4-msec RTS on Day 1 is shown for comparison.

not reach significance [Group 1, Day 1 vs. Day $3, t(11)=$ $1.47, p>.15$; Group 1, Day 1 vs. Group 3, Day $3, t(22)=$ $1.16, p>.20]$.

Training with either a $0.4-\mathrm{msec}$ RTS or a $300-\mathrm{msec}$ RTS appeared to have the same effect on LTH when rats were tested with a $0.4-\mathrm{msec}$ RTS. On Day 2, Group 2 showed significant LTH following training with the 300 msec RTS, and Group 1 and Group 3 showed an almost identical course of responding over trials on Day 3. Clearly, training with the $300-\mathrm{msec}$ RTS significantly affected responding to the $0.4-\mathrm{msec}$ RTS when testing was over days rather than within sessions. However, the purest measure of LTH is the response on the first trial of a daily session, and these first-trial comparisons were not significant on Day 3. Single-trial startle data are notoriously variable, and it was largely that variability that prevented the single-trial data from reaching significance. If 2 animals that were more than two standard deviations above the mean of their distributions were removed from the analysis, one from Group 1 and one from Group 3, the relevant first-trial comparisons yielded significant results [Group 1, Day 1 vs. Day 3, $t(10)=2.31, p<.05$; Group 1, Day 1 vs. Group 3, Day 3, $t(20)=2.32, p<.05]$.

These startle data strongly suggest that, whereas STH is inversely related to stimulus rise time and directly related to the degree of response evocation, LTH is relatively independent of these variables. A stimulus that produced little or no response and no detectable STH pro- duced robust response decrements over days. Thus, these data yield the surprising result of apparently showing LTH in the absence of STH.

Freezing and vocalization. Procedural limitations only allowed the sampling of freezing and vocalization from one of the four test chambers. Thus, we have such data only from 3 rats from each group, and the results must be appropriately qualified. The results are summarized in Figure 4. (The Day 1 comparisons combined Groups 2 and 3, but only Group 3 is shown on subsequent days.) Both the 0.4-msec RTS and the 300-msec RTS provoked freezing (Figure 4A) and vocalization (Figure 4B) on Day 1. The 0.4-msec stimulus was more potent in this regard, provoking significantly more freezing $[t(7)=2.65$, $p<.05]$ and vocalization $[t(7)=3.12, p<.05]$ than the $300-\mathrm{msec}$ RTS in Groups 2 and 3 combined.

Freezing and vocalization declined over days, reaching a low level for both Group 1 and Group 3 on Day 3, when both groups received the 0.4-msec RTS. These groups did not differ on Day 3 on either measure $(t \mathrm{~s}<$ 1). However, Group 3 showed significantly less freezing $[t(4)=5.41, p<.01]$ and vocalization $[t(4)=3.30, p<$ $.05]$ on Day 3 than did Group 1 on Day 1.

These emotional responses to the startle-inducing stimuli, like the ASR, are sensitive to the rise time of the acoustic stimulus. Even though there was more acoustic energy in the 300-msec RTS than in the 0.4-msec RTS, the shorter RTS produced significantly more freezing and 

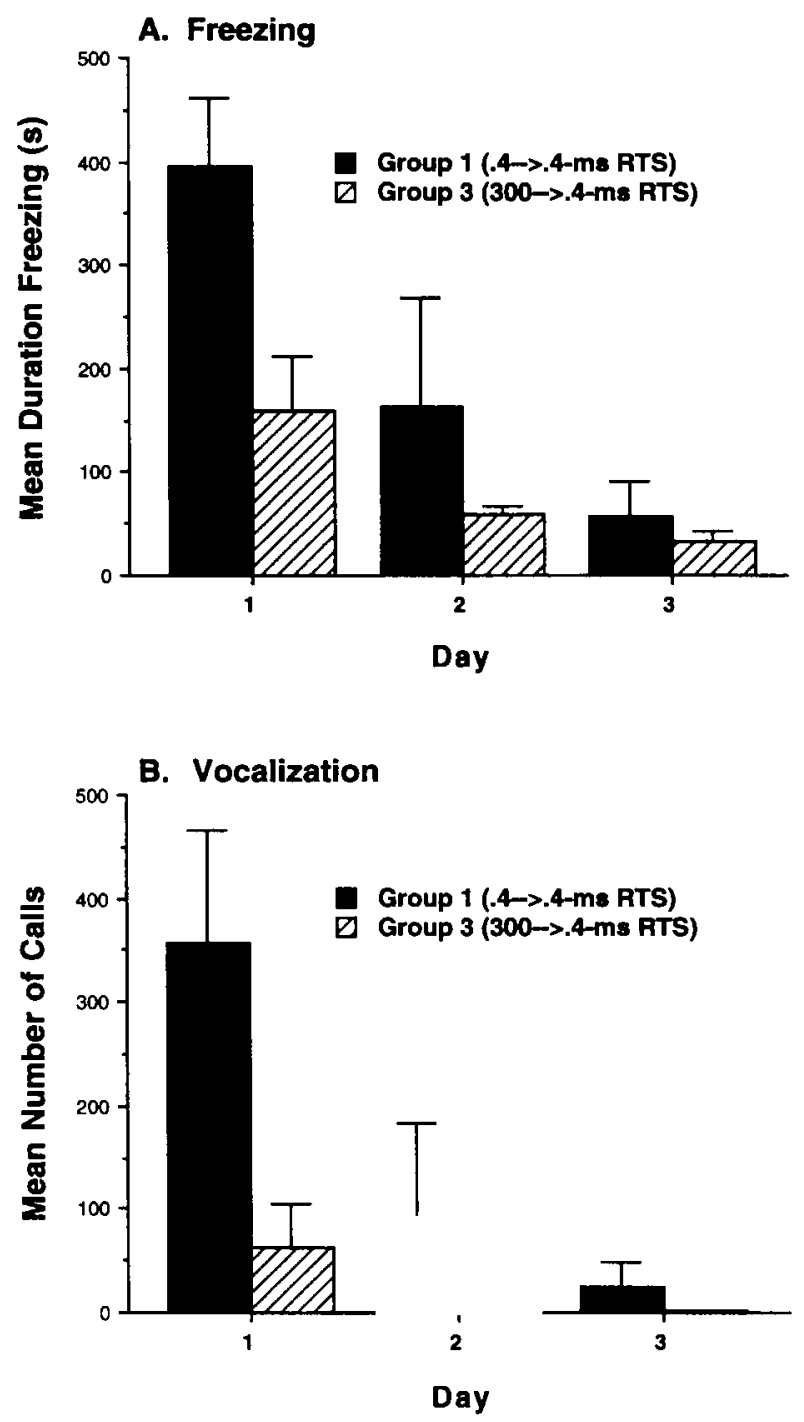

Figure 4. Mean duration of freezing ( $A$ ) and mean number of vocalizations (B) over the 3 days in Experiment 1. Group $1(n=$ 3) received a $0.4-\mathrm{msec}$ rise time stimulus (RTS) on all days. Group $3(n=3)$ received a 300-msec RTS on Days 1 and 2 and a 0.4-msec RTS on Day 3. (Groups 2 and 3 were treated identically on Day 1 and are combined in the figure $[n=6]$. Only Group 3 is shown on Days 2 and 3.) Error bars are standard errors.

vocalization on Day 1. However, experience with the 300-msec RTS reduced the potential for the $0.4-\mathrm{msec}$ RTS to induce freezing and vocalization and did so, apparently, as effectively as did experience with the $0.4-\mathrm{msec}$ RTS itself. In this regard, the effect appears to be similar to the effects on the ASR itself: A stimulus that produced little initial response effectively reduced responsiveness to the more potent stimulus.

\section{EXPERIMENT 2}

In Experiment 1, the effects of stimulus rise time on STH was shown by testing the animals with one un- changed condition (0.4-msec RTS to 0.4-msec RTS) and one changed condition (300-msec RTS to $0.4-\mathrm{msec}$ RTS). It is possible that the change itself contributed to the greater responsiveness in the changed than in the unchanged condition. Experiment 2 tested this possibility by training animals with either a fast or a slow RTS and then testing for STH at an intermediate rise time value.

\begin{abstract}
Method
Subjects and apparatus. Thirty of the rats from Experiment 1 were used. Four were excluded because they were used in preliminary testing, and 2 because of apparatus failure. The apparatus was identical to that used in Experiment 1 . The intensity of the $10-\mathrm{kHz}$ stimulus was increased to $107-\mathrm{dB}$ SPL. Three rise time values were used: $2 \mathrm{msec}, 12 \mathrm{msec}$, and $72 \mathrm{msec}$. These values represent approximately equal log steps, and preliminary testing showed that the 12-msec RTS would produce an adequate response level. Stimulus duration was $100 \mathrm{msec}$ plus the rise/fall time; thus, each stimulus stayed at its maximum intensity for $100 \mathrm{msec}$. Startle amplitude was taken as the difference between the maximum voltage for $152 \mathrm{msec}$ (i.e., $80 \mathrm{msec}$ plus the slowest rise time) before and after stimulus onset.

Procedure. All the animals were tested in two sessions. In each session, each rat received 40 stimulus presentations on a 20 -sec ISI. For the first 20 trials of a session, the stimulus was either the 2msec RTS or the 72-msec RTS. For the second 20 trials, the stimulus was always the 12 -msec RTS. Half of the rats received the 2- to 12-msec sequence in Session I and the 72- to 12-msec sequence in Session 2. The other half received the sequences in reverse order. The order of presentation was balanced by the rats' group assignments in Experiment 1.
\end{abstract}

\section{Results}

There were no significant order effects $[F(1,28)=$ $1.23, p>.25]$, so the results of Sessions 1 and 2 were combined for analysis. Figure 5 summarizes these data for the 10 test trials following training with the two different RTS. Startle amplitude was significantly lower to the 12-msec RTS following training with the 2-msec RTS than following training with the $72-\mathrm{msec}$ RTS $[t(29)=$ $3.16, p<.01]$. Therefore, responsiveness in the test series depended on the rise time of the stimulus in the training series and not simply on the change in rise time from the training to the test series.

\section{EXPERIMENT 3}

The surprising outcome of Experiment 1 was that LTH of acoustic startle appeared to be independent of stimulus rise time and, therefore, independent of the degree of response evocation. Rats that had received 40 presentations of a $300-\mathrm{msec}$ RTS were no more responsive to a $0.4-$ msec RTS when it was first presented than were rats that had received the 0.4-msec RTS in all the sessions. However, during training, the 300-msec RTS group was exposed to the general test conditions as well as to the slowrise stimuli, and that general exposure could have contributed to the test-day response deficit.

Experiment 3 was a partial replication of Experiment 1 , in an attempt to add the strength of replication to the surprising LTH result and to control for any contri- 


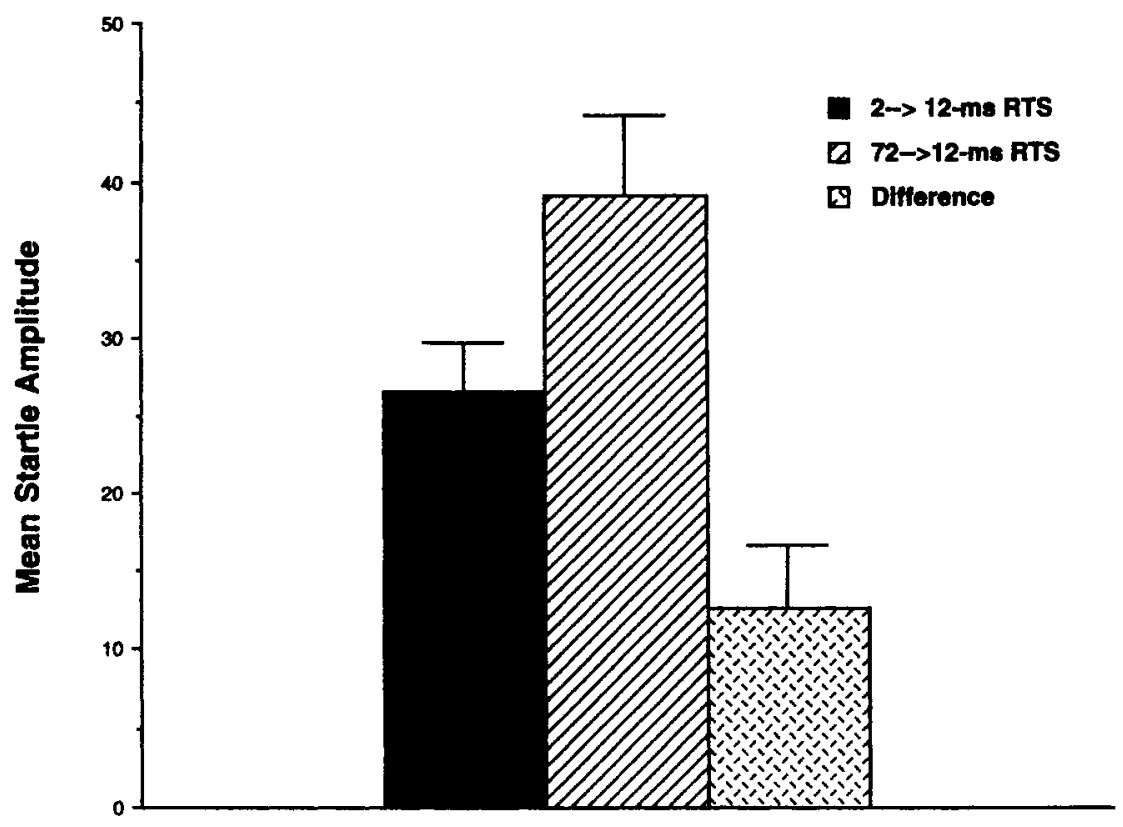

Figure 5. Mean startle amplitude to a 12-msec rise time stimulus (RTS) after 20 trials with a 2-msec RTS or after 20 trials with a 72 -msec RTS and the mean difference in Experiment 2. Error bars are standard errors $(n=30)$.

bution made to the response deficits by general exposure to the training conditions. One group received daily presentations of a 0.4-msec RTS; a second group received daily presentations of a 300-msec RTS; and a third group simply received daily placements in the startle chamber. All the groups received the $0.4-\mathrm{msec}$ RTS on the test day. Additional training days were used, in an attempt to optimize LTH, and the design required a between-group comparison to assess LTH.

In addition to the basic replication, Experiment 3 was run in a different laboratory, with the hope of adding an interlaboratory dimension of robustness to the phenomena. The first two experiments were run in the first author's laboratory at the University of Tübingen. Experiment 3 was run in the second author's laboratory at Dartmouth College.

\section{Method}

Subjects. Thirty-three male Long-Evans-derived (hooded) rats, born and raised in the Dartmouth College psychology department vivarium, were individually housed and maintained on a 14:10-h light:dark cycle. They were approximately 130 days old at the beginning of the experiment and weighed between 430 and $600 \mathrm{~g}$. They had free access to food and water throughout the experiment. Testing took place between 15:00 and 18:00.

Apparatus. The startle apparatus was a modification of one described in detail previously (Leaton, 1976). The animals were tested in one of two holding cages $(20 \times 12 \times 14 \mathrm{~cm})$ fabricated from aluminum, except for one Plexiglas end wall and a floor consisting of 2.5-mm brass rods fixed to Phenolite insulating material. Each cage was sandwiched between compression springs attached to a rigid superstructure, housed within a separate sound-attenuating chamber, and illuminated by a $7-W$ incandescent bulb. Vertical displacement of the cage moved an attached magnet within a fixed coil, inducing a voltage that was amplified and then digitized and analyzed by a microcomputer system. Startle amplitude was taken to be the maximum voltage for a fixed time window after stimulus onset. Both 80- and 380-msec time windows were recorded on each trial. Small video cameras (Watec, WAT-660-D, Edmund Scientific) allowed continuous observation of the animals on remote monitors.

The startle stimuli were nominally the same as those in Experiment $1-10-\mathrm{kHz}, 105-\mathrm{dB}$ tone bursts delivered by a $9-\mathrm{cm}$ piezoelectric tweeter (Herald Electronics) centered $12 \mathrm{~cm}$ from the long wall of the cage. Background white noise of 50-dB SPL was delivered continuously by a $10-\mathrm{cm}$ speaker mounted above the tweeter. Auditory intensities were measured with a Quest impulse soundlevel meter (Model 2700, A scale, max) with the microphone centered inside the startle chamber. The slow-rise stimulus had a 300-msec rise/fall time and was $700 \mathrm{msec}$ in duration; the fast-rise stimulus had a 0.4-msec rise/fall time and was $100.8 \mathrm{msec}$ in duration.

Procedure. The rats were divided into three groups of 11 matched for body weight, and each rat was handled for 1-2 min each day for 4 days. They were then given five daily 8 -min preexposure sessions in the startle chamber with no auditory stimulation other than the background white noise. These sessions were followed by four daily training sessions and then one test session. During each training session, Group Fast received 40 presentations of the 0.4-msec RTS, and Group Slow received 40 presentations of the 300 -msec RTS. Group No was simply placed in the startle chamber for a standard session time, but with no startle stimulus presentation. "Trials" were run for Group No with the startle stimulus speakers turned off, so that background activity was measured on each trial. On the test session, all the groups received 20 presentations of the $0.4-\mathrm{msec}$ RTS. During each session, all the stimuli were presented on a $20-\mathrm{sec}$ ISI, and the first stimulus was presented after 5 min. Freezing, as defined in Experiment 1, was sampled from each rat every $15 \mathrm{sec}$, beginning with the first startle 


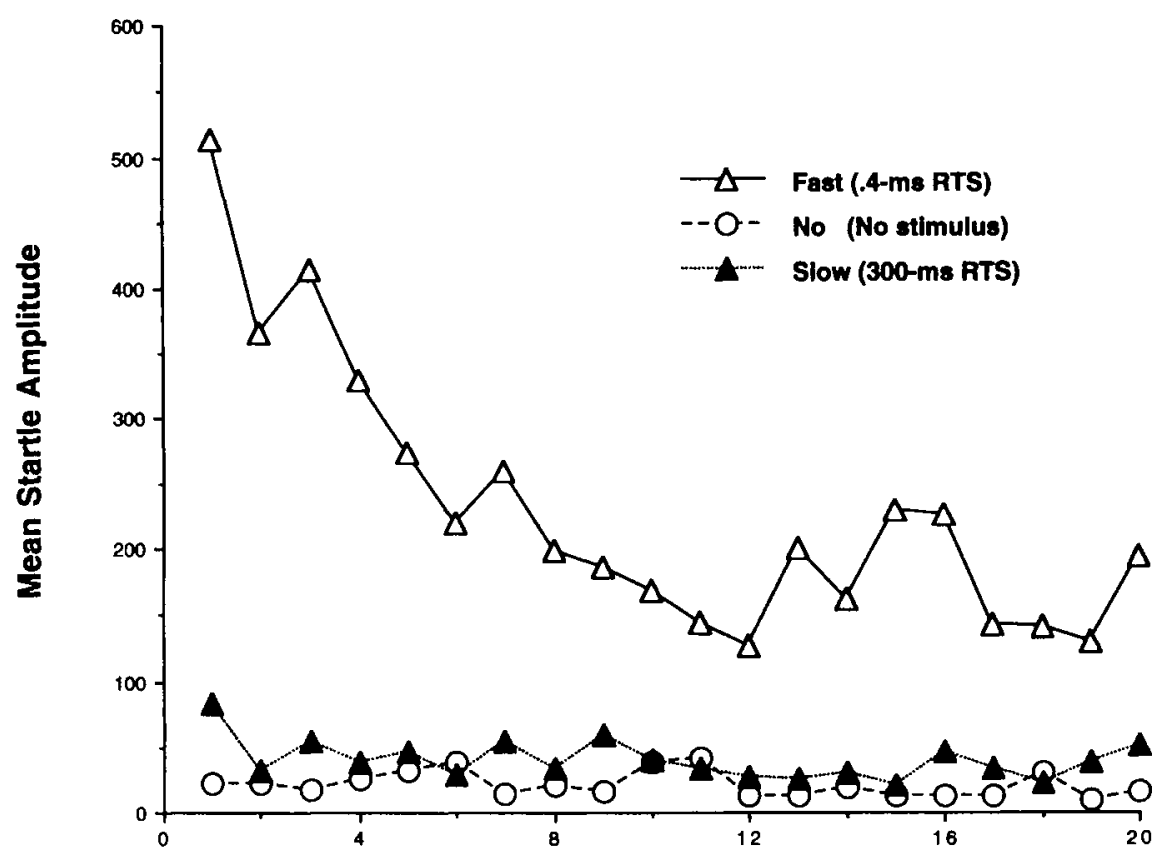

Trial Number

Figure 6. Mean startle amplitude to a 0.4-msec rise time stimulus (RTS; Fast, $n=11$ ) or a 300-msec RTS (Slow, $n=11)$ on the first training day in Experiment 3. No $(n=11)$ indicates the baseline response level for the group that received no startle stimuli.

stimulus and continuing for $6 \mathrm{~min}$. In this way, 24 time samples were judged as either freezing or not freezing, and a percentage freezing score was calculated.

The rats were run in two squads. Squad 1 consisted of 6 rats per group, with an average weight of $490 \mathrm{~g}$. Squad 2 ( 5 rats per group, average weight $525 \mathrm{~g}$ ) began the experiment as soon as Squad 1 had completed it. The squads were drawn from the same original pool of 33 rats, the results for the two squads did not differ, and they were combined for all analyses. Statistical analyses followed the same protocols as those described in Experiment 1. All the sessions were run on consecutive days.

\section{Results and Discussion}

Startle amplitude. The results for the first training day for the 380 -msec response window are shown in Figure 6 . The results for the fast and slow rise time groups basically replicated the results of Experiment 1 . The fast RTS produced significantly more response than did the slow RTS. Analysis of these two groups over the first 10 trials showed a significant difference between groups $[F(1,20)=25.41, p<.001]$. The slow RTS did produce some response in the $380-\mathrm{msec}$ window. There was a significant difference between Group Slow and Group No over the first 10 trials $[F(1,20)=10.54, p<.001]$.

As in Experiment 1, the responses to the 0.4-msec RTS in the 80-msec and 380-msec response windows were nearly identical. The $300-\mathrm{msec}$ RTS produced no detectable response in the 80-msec window, and Group Slow did not differ from Group No in this response window $[t(20)=1.12, p>.20]$. For all further analyses, only the $380-\mathrm{msec}$ response window will be reported.

Long-term habituation of startle. The startle response data for the test day, Day 5, are shown in Figure 7. All the groups were tested under identical conditions with the 0.4-msec RTS on this day. Again replicating Experiment 1, Group Fast and Group Slow showed very similar response levels over all trials. Neither the firsttrial difference $[t(20)=1.08, p>.20]$ nor the difference over trials $1-10(F<1)$ was significant, and comparisons over Trial Blocks $2,3,4$, or 5 did not approach significance (all $F \mathrm{~s}<1$ ). This pattern of statistical results is identical to the results of the comparable comparisons in Experiment 1.

A one-way ANOVA for Trial 1 alone for all three groups yielded a marginally significant group difference $[F(2,30)=3.21, p=.055]$, and post hoc analysis (Bonferroni) showed only that Group Fast was significantly different from Group No $(p<.01)$. However, an ANOVA over Trials 1 and 2 yielded a significant overall group difference $[F(2,30)=5.09, p<.02]$ and significant differences between Group Fast and Group No $[F(1,20)=$ $7.89, p<.02]$ and between Group Slow and Group No $[F(1,20)=4.75, p<.05]$. Group No showed an unexpected sensitization-like effect that elevated response amplitudes from Trials $3-5$ and contributed to the continuing 


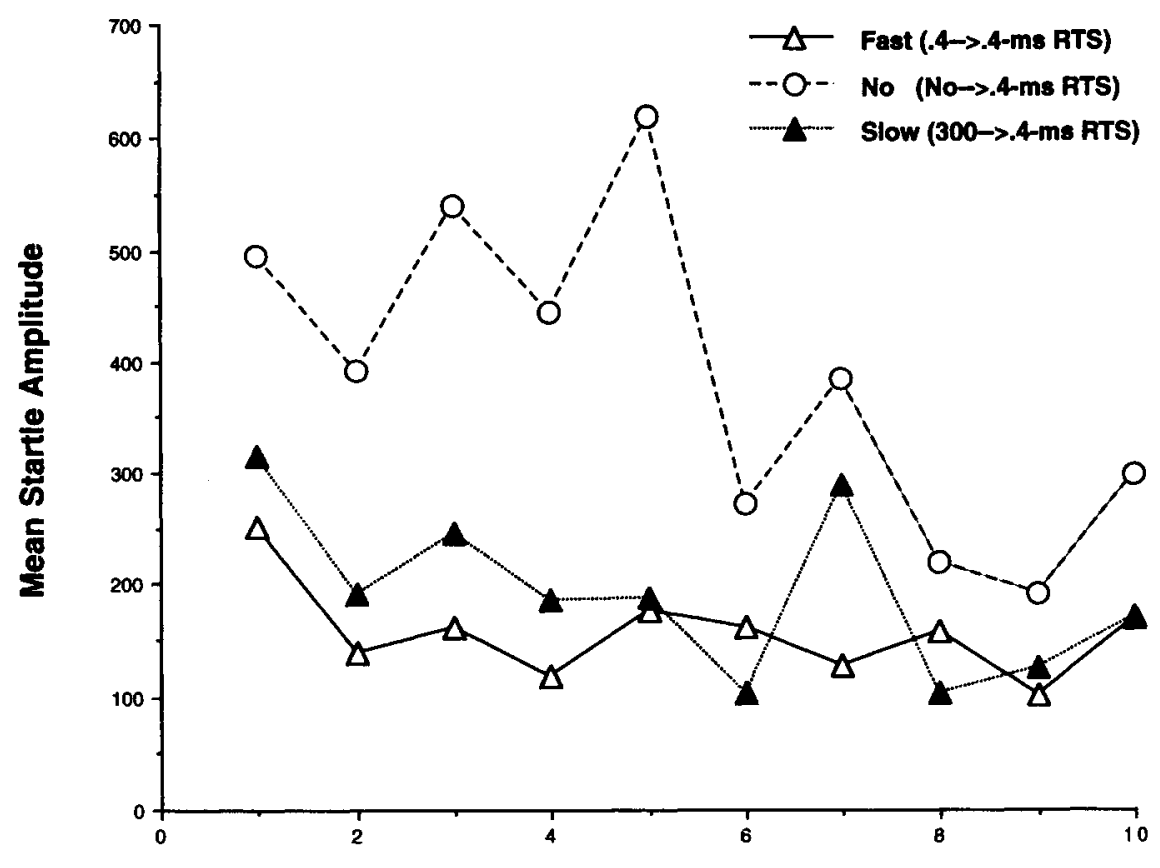

Trial Number

Figure 7. Mean startle amplitude to a 0.4-msec rise time stimulus (RTS) on the test day (Day 5) in Experiment 3. On each of 4 previous days, Group Fast $(n=11)$ had received 40 trials with a 0.4-msec RTS, Group Slow $(n=11)$ had received 40 trials with a 300 -msec RTS, and Group No $(n=11)$ had simply been placed in the startle chamber without stimulus presentations.

difference between Group No and the other two groups over these trial blocks [smallest $F \mathrm{~s}(1,20)=7.89$ and 4.73, $p s<.05$, for Group Fast and Group Slow respectively].

As in Experiment 1, training with a 300-msec RTS significantly affected responding to a $0.4-\mathrm{msec}$ RTS over days. However, also as in Experiment 1, the first trial differences, the purest measure of LTH, did not reach significance for the comparison between Group Slow and Group No. These data may indicate that the fast RTS produced somewhat more LTH than did the slow RTS, but the differences over trials between Group No and the other two groups clearly showed that experience with the 300-msec RTS produced significant long-term response decrements to the 0.4-msec RTS and that simply time in the test chambers did not contribute significantly to these response decrements.

Freezing. The data for the freezing measure for the three groups are shown in Figure 8. (Group No was not included in the analysis of training sessions, since that group showed little or no freezing on those days.) Once again replicating the Experiment 1 results, the fast RTS produced significantly more freezing than did the slow RTS on Day $1[t(20)=2.52, p<.05]$ and over the four training sessions $[F(1,20)=6.36, p<.05]$. Freezing decreased over days for both groups $[F(1,20)=38.87, p<.01]$.

On the test session, Day 5, the three groups differed significantly $[F(2,30)=10.54, p<.01]$. Post hoc Bonfer- roni comparisons showed that both Group Fast $(p<.01)$ and Group Slow $(p<.05)$ differed from Group No, but Groups Fast and Slow were not significantly different $(p=$ .45). As in Experiment 1, experience with the slow RTS reduced the potential for the fast RTS to induce freezing: On Day 5, Group Slow froze significantly less than did Group Fast on Day $1[t(20)=2.79, p<.02]$. However, Group Slow did show increased freezing from Day 4 to Day 5 . This increase was only marginally significant $[t(10)=2.16, p=.06]$, but it suggests that the fast RTS was somewhat better in reducing freezing than was the slow RTS, even though the slow RTS contains more acoustic energy.

\section{GENERAL DISCUSSION}

In the present experiments, the amplitude of the ASR and STH of the response depended critically on the rise time of the startle-inducing stimulus. Both amplitude and STH were inversely related to rise time. In contrast to STH, LTH of the ASR appeared to be largely independent of the response and of stimulus rise time. A $300-\mathrm{msec}$ RTS that produced little or no response and no detectable STH nonetheless produced significant response decrements over days. Fear-like emotional responses, freezing, and vocalization were induced by both the fast- and the slow-rise stimuli, but the fast-rise stimuli were more po- 


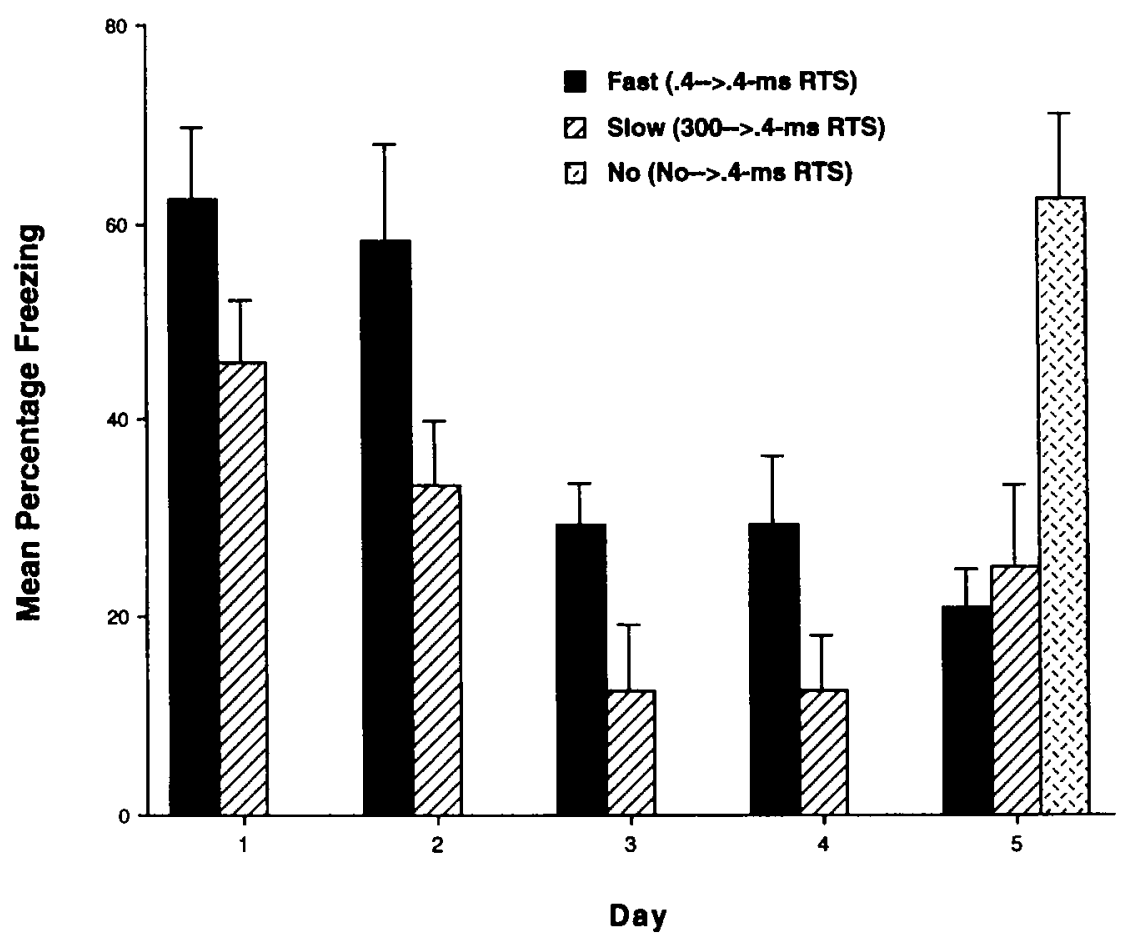

Figure 8. Mean percentage freezing for the three groups over the 5 days in Experiment 3. On each of Days $1-4$, Group Fast $(n=11)$ received 40 presentations of a $0.4-\mathrm{msec}$ rise time stimulus (RTS), Group Slow $(n=11)$ received 40 presentations of a 300 -msec RTS, and Group No $(n=11)$ received no startle stimulus presentations and showed little or no freezing. All the groups received 20 presentations of a 0.4-msec RTS on Day 5. Error bars are standard errors.

tent in this regard. These emotional responses habituated over days, and exposure to slow-rise stimuli effectively reduced the emotional responses subsequently induced by fast-rise stimuli.

\section{Short-Term Habituation}

STH of ASR is indexed by decreased responsiveness to repeated auditory stimulation within a session. In Experiment 1 , a $300-$ msec RTS produced little or no response and did not produce any detectable STH when the stimulus was changed within a test session to a $0.4-\mathrm{msec}$ RTS. Experiment 2 controlled for the simple factor of stimulus change and showed that, when testing was to a common 12-msec RTS, there was more response (i.e., less STH) following training with a 72-msec RTS than following training with a 2-msec RTS. These data confirm a preliminary report (Pilz, 1989) in showing that STH of the ASR is inversely related to the rise time of the startleeliciting stimulus.

STH is assumed to be an intrinsic process in which the cellular changes that produce the response decrements occur as synaptic depression at some site within the startle response circuit itself. The data that support this assumption are indirect, consisting primarily of the failure of physiological manipulations, such as brain lesions or drug injections, to influence STH without at the same time significantly affecting the response itself (Davis \& File, 1984). The demonstrated inverse relation between stimulus rise time and STH is consistent with the assumption that STH is an intrinsic process.

If this assumption is correct, STH must depend critically on the degree of activation of neurons in the primary startle circuit. Startle amplitude and the amplitude threshold of the response should both be rather direct reflections of the degree of activation of the startle circuit, and both are inversely related to stimulus rise time (Fleshler, 1965; Pilz, 1989). At the neural level, activation of neurons within the PnC reflect many of the behavioral characteristics of the ASR, and their firing thresholds are inversely related to stimulus rise time (Lingenhöhl \& Friauf, 1994; Wu et al., 1988). These neurons apparently are a critical part of the ASR circuitry and are generally considered to be the sensorimotor interface for the ASR. However, the degree of activation specifically of the PnC neurons may not be directly related to STH. Startle amplitude is reduced if a relatively weak stimulus-for example, a low-intensity tone - precedes the startle-eliciting stimulus by $100 \mathrm{msec}$ or so (Hoffman \& Ison, 1980). The firing rate of neurons in the $\mathrm{PnC}$ is reduced by an auditory prepulse (Wu et al., 1988). In one experiment (Hoffman, Cohen, \& Corso, 1984), STH was not affected by the reduction in startle amplitude produced by an auditory pre- 
pulse. The present data combined with these prepulse data suggest that STH occurs within the startle circuit prior to PnC and prior to the site of the inhibitory action of a prepulse. It is, of course, possible that STH is an extrinsic process, but if it is, the extrinsic circuit must require the same rise time parameters for activation as does the startle circuit itself.

Not only was STH inversely related to stimulus rise time, but the 300-msec RTS used in Experiment 1 produced no detectable STH. However, this stimulus did produce a detectable response on Day 1 in Experiments 1 and 3. This response was not detected in the $80-\mathrm{msec}$ response window, appearing only in the $380-\mathrm{msec}$ response window. The primary startle response has a latency of $8-10 \mathrm{msec}$ when measured in the hindlimb muscles (Davis et al., 1982; Szabo, 1965) and a latency of less than $20 \mathrm{msec}$ when measured at a transducing platform similar to the one used here (Pilz \& Schnitzler, 1996). In the critical first $12 \mathrm{msec}$ of a potentially startle-inducing stimulus (Fleshler, 1965), the 300-msec RTS had only reached $77 \mathrm{~dB}, \mathrm{SPL}$. This value is well below the typically reported value for the threshold of the ASR with a 10$\mathrm{kHz}$ stimulus (Fleshler, 1965; Pilz et al., 1987). These considerations make it unlikely that the responses to the 300-msec RTS represented activity in the startle circuit. In a video analysis of the rat's responses to auditory stimuli of various rise times, Oedekoven (1996) identified body and head movement responses with latencies longer than $80 \mathrm{msec}$ that were clearly distinct from the startle response itself. Our transducer would have been sensitive to such movements, and it was probably these responses, rather than startle per se, that produced a response in the $380-\mathrm{msec}$ window that was not seen in the $80-\mathrm{msec}$ window. Although we cannot completely rule out the possibility that the 300-msec RTS produced some smallamplitude, infrequent, short-latency responses that represented neural activity in the primary startle circuit, such responses were not adequate to produce STH.

\section{Long-Term Habituation}

In both Experiments 1 and 3, experience with a 300msec RTS, a stimulus that produced little or no startle response and no detectable STH, produced significant response decrements over days when tested with a 0.4msec RTS. These data show that STH and LTH of the ASR are sensitive to different characteristics of the acoustic stimulus. STH was inversely related to stimulus rise time and, thus, directly related to response amplitude. By contrast, LTH appeared to be independent of stimulus rise time and, thus, independent of response amplitude. Our procedures may not have been sensitive enough to detect small differences in LTH between the fast and the slow RTS. However, there seems to be no question that, if LTH is sensitive to the rise time variable at all, it is much less so than is STH.

Both behavioral and physiological data suggest that LTH and STH are independent processes that require different underlying neural mechanisms (Jordan, 1989;
Leaton, 1976; Leaton \& Supple, 1986, 1991). Such data require that $\mathrm{LTH}$ occur in a neural circuit that is extrinsic to the primary startle circuit, in contrast to the presumed intrinsic nature of STH. The present data are consistent with this position and suggest that the LTH circuitry does not depend on all of the same stimulus parameters for activation as does the startle circuitry itself. Given the parametric characteristics of neurons in the PnC (Lingenhöhl \& Friauf, 1994; Wu et al., 1988), it seems unlikely that they were activated by the $300-\mathrm{msec}$ RTS, and our procedures could not detect startle to these stimuli. Although the LTH and STH circuitry must overlap at some point on the sensory side of PnC, the LTH circuitry is activated by stimuli that do not activate $\mathrm{PnC}$ or elicit the response.

Habituation of sensitization could contribute to the response decrements over days that we have described as LTH of startle. According to the two-factor theory of habituation (Groves \& Thompson, 1970; Thompson \& Spencer, 1966), presentation of a startle stimulus induces two processes: a response-decreasing process (habituation) and a response-increasing process (sensitization). The actual measured response is assumed to reflect the summation of these two processes. With repeated stimulation, the startle response itself habituates and so, too, does sensitization. Response decrements over days, then, can represent both the habituation of the startle response itself and habituation of sensitization. Applying these conceptualizations to the LTH data of Experiments 1 and 3 , we assume that the initial Day 1 responsiveness to the fast RTS, after the very first trial, is elevated by the sensitizing effects of the acoustic stimulus itself. This sensitization habituates with repeated testing, so that the response decrements from the first day to the test day may reflect, at least in part, habituation of sensitization. The first trial of the test day should reflect LTH of the startle response itself, but subsequent trials may be reduced in amplitude, as compared with the first day, because of habituation of the inflating effects of sensitization. Although first-trial responsiveness for the slow RTS groups was reduced on the test days in both Experiment 1 and Experiment 3 ; these differences did not reach statistical significance. In both cases, adding Trial 2 to the comparisons produced statistical significance. This second trial served to stabilize the inherently variable single-trial startle data, but, of course, it also introduced the possibility of a contribution from the habituation of sensitization.

\section{Freezing and Vocalizations}

The acoustic stimuli used in these experiments provoked freezing and vocalization, and we have suggested that these emotional responses represent a fear-like sensitization process that is a routine feature of the standard ASR paradigm (Borszcz, Cranney, \& Leaton, 1989; Leaton \& Cranney, 1990; Plappert, Pilz, \& Schnitzler, 1993). Both the slow and the fast RTS induced freezing (Experiments 1 and 3 ) and vocalization (Experiment 1), and like the startle response itself, these emotional responses were sensitive to stimulus rise time and decreased 
over test days. Experience with the slow RTS significantly reduced the emotional responses induced by the more potent fast RTS. If these responses index a startleenhancing sensitization process, as we assume they do, their habituation over days could have contributed to the apparent LTH produced by the 300 -msec RTS. These emotional responses may provide a measure of the sensitization process discussed above.

Two possible confounding issues should be addressed. First, we chose to use pure tone stimuli in these experiments, rather than noise, to maintain consistency with previous data (Pilz, 1989) and because the rise times of pure tones are more easily controlled. However, the frequency characteristics of the various stimuli used in these experiments may have varied as a function of rise time. The sudden onset of a pure tone produces a complex waveform that can be detected as an audible click, and this acoustic splatter could have produced spectral differences between our fast and slow RTS. In unpublished observations, Pilz (1998) performed a Fourier spectrum analysis and narrow band frequency analysis on a $105-\mathrm{dB}, 0.4-$ msec RTS with an 8-msec duration. The 8-msec duration was a conservative choice, because the energy distribution becomes worse when the time duration is shortened. This analysis showed that $95 \%$ of the energy (rms) of the stimulus fell within the region $10 \mathrm{kHz} \pm 100 \mathrm{~Hz}$. The frequency discrimination in the rat at $10 \mathrm{kHz}$ is estimated to be about $300 \mathrm{~Hz}$ (Gourevitch, 1965). In addition, Pilz (1998) varied the rise time of a $20-\mathrm{msec}, 108-\mathrm{dB}, 10-\mathrm{kHz}$ tone through the values of $0,0.1,0.2,0.4,0.8,1.6,3.2$, 5.0 , and $6.4 \mathrm{msec}$. Both the spectral analysis and the subjective response of human listeners revealed strong acoustic splatter for the 0 and the 0.1 RTS but not for the longer RTS. These observations make us confident that the rise time differences in the stimuli, and not differences in their spectral characteristics, account for their different experimental effects.

Second, the durations of the fast- and slow-rise stimuli were different. The slow RTS was always longer in duration than the fast RTS, and therefore, the slow RTS always contained more acoustic energy than the fast RTS. In spite of the energy differential, the slow RTS did not produce detectable STH, and it was less effective than the fast RTS in inducing freezing and vocalization. One might guess that the difference in freezing would have been even greater had we equated stimulus energy, and the greater energy in the slow RTS may have contributed to the reduction in freezing when testing with the fast RTS. It is also possible that the greater acoustic energy in the slow RTS contributed to the LTH of the startle response. Of course, it would have had this LTH effect in neural elements that were not activated adequately to produce the response.

The data from these three experiments make it clear that response decrements over days in the ASR are relatively independent of stimulus rise time and, thus, of the response amplitude during training. In contrast, within- session response decrements, which define STH, depend critically on stimulus rise time. The response decrements over days may represent both LTH of the ASR itself and LTH of sensitization. It is possible that the rise time variable influenced these LTH effects but that our procedures were not sensitive enough to detect the relatively small effects. Whatever the case, it is clear that LTH is much less sensitive to the rise time variable than is STH. These data are consistent with the view that LTH occurs within a neural circuitry that is extrinsic to the basic startle circuitry and that STH occurs within some element of the basic circuitry. We believe that these processes can best be conceptualized by assuming at least three independent neural circuits: (1) an intrinsic STH startle circuit, (2) an extrinsic LTH startle circuit, and (3) an extrinsic sensitization circuit.

\section{REFERENCES}

Borszcz, G. S., Cranney, J., \& Leaton, R. N. (1989). Influence of long-term sensitization on long-term habituation of the acoustic startle response in rats: Central gray lesions, preexposure, and extinction. Journal of Experimental Psychology: Animal Behavior Processes, 15, 54-64.

DAVIS, M., \& FILE, S. E. (1984). Intrinsic and extrinsic mechanisms of habituation and sensitization: Implications for the design and analysis of experiments. In H. V. S. Peeke \& L. Petrinovich (Eds.), Habituation, sensitization, and behavior (pp. 287-323). San Diego: Academic Press.

Davis, M., Gendelman, D. S., Tischler, M. C., \& Gendelman, P. M (1982). A primary acoustic startle circuit: Lesion and stimulation studies. Journal of Neuroscience, 2, 791-805.

FANSELOW, M. S. (1980). Conditional and unconditional components of post-shock freezing. Pavlovian Journal of Biological Science, 15(4), 177-182.

FLESHLER, M. (1965). Adequate acoustic stimulus for startle reaction in the rat. Journal of Comparative \& Physiological Psychology, 60, 200-207.

GouREvITCH, G. (1965). Auditory masking in the rat. Journal of the Acoustical Society of America, 37, 439-443.

Groves, P. M., \& THOMPSON, R. F. (1970). Habituation: A dual-process theory. Psychological Review, 77, 419-450.

Hoffman, H. S., Cohen, M. E., \& Corso, C. (1984). Reflex modification during habituation of a startle response. Bulletin of the Psychonomic Society, 22, 574-576.

Hoffman, H. S., \& Ison, J. R. (1980). Reflex modification in the domain of startle: I. Some empirical findings and their implications for how the nervous system processes sensory input. Psychological Review, 87, 175-189.

JoRDAN, W. P. (1989). Mesencephalic reticular formation lesions made after habituation training abolish long-term habituation of the acoustic startle response in rats. Behavioral Neuroscience, 103, 805-815.

KAltWASSER, M. T. (1990). Startle-inducing acoustic stimuli evoke ultrasonic vocalization in the rat. Physiology \& Behavior, 48, 13-17.

Kandler, K., \& Herbert, H. (1991). Auditory projections from the cochlear nucleus to pontine and mesencephalic reticular nuclei in the rat. Brain Research, 562, 230-242.

Koch, M., LingenhöHL, K., \& Pilz, P. K. D. (1992). Loss of the acoustic startle response following neurotoxic lesions of the caudal pontine reticular formation: Possible role of giant neurons. Neuroscience, 49, 617-625.

LEATON, R. N. (1976). Long-term retention of the habituation of lick suppression and startle response produced by a single auditory stimulus. Journal of Experimental Psychology: Animal Behavior Processes, 2, 248-259.

Leaton, R. N., \& Cranney, J. (1990). Potentiation of the acoustic star- 
tle response by a CS paired with an acoustic startle stimulus in rats. Journal of Experimental Psychology: Animal Behavior Processes, $16,279-287$.

LEATON, R. N., \& SUPPLE, W. F., JR. (1986). Cerebellar vermis: Essential for long-term habituation of the acoustic startle response. Science, 232, 513-515.

Leaton, R. N., \& SupPle, W. F., JR. (1991). Medial cerebellum and long-term habituation of acoustic startle in rats. Behavioral Neuroscience, 105, 804-816.

Lee, Y., Lopez, D. E., Meloni, E. G., \& Davis, M. (1996). A primary acoustic startle pathway: Obligatory role of cochlear root neurons and the nucleus reticularis pontis caudalis. Journal of Neuroscience, 16, 3775-3789.

LingENHöhl, K., \& Friauf, E. (1994). Giant neurons in the rat reticular formation: A sensorimotor interface in the elementary acoustic startle circuit? Journal of Neuroscience, 14, 1176-1194.

Marsh, R., Hoffman, H. S., \& STITT, C. L. (1973). Temporal integration in the acoustic startle reflex of the rat. Journal of Comparative \& Physiological Psychology, 82, 507-511.

OEDEKOVEN, C. (1996). Akustisch initierte Verhaltens- und Herzratenänderungen bei der Laborratte [Acoustically induced changes in behavior and heart rate in laboratory rats]. Unpublished doctoral dissertation, University of Tübingen.

PILz, P. K. D. (1989). Die akustisch ausgelöste Schreckreaktion bei der Ratte [The acoustically triggered startle response in the rat]. Unpublished doctoral dissertation, University of Tübingen.
PiLz, P. K. D. (1998). [Spectral characteristics of pure tones as a function of stimulus rise time.] Unpublished raw data.

Pilz, P. K. D., \& Schnitzler, H.-U. (1996). Habituation and sensitization of the acoustic startle response in rats: Amplitude, threshold, and latency measures. Neurobiology of Learning \& Memory, 66, 67-79.

Pilz, P. K. D., Schnitzler, H.-U., \& Menne, D. (1987). Acoustic startle threshold of the albino rat (Rattus norvegicus). Journal of Comparative Psychology, 101, 67-72.

Plappert, C. F., Pilz, P. K., \& Schnitzler, H.-U. (1993). Acoustic startle response and habituation in freezing and nonfreezing rats. $B e-$ havioral Neuroscience, 107, 981-987.

SzABo, I. (1965). Analysis of the muscular action potentials accompanying the acoustic startle reaction. Acta Physiologica Hungarica, 27, 167-178.

Thompson, R. F., \& SPEncer, W. A. (1966). Habituation: A model phenomenon for the study of neuronal substrates of behavior. Psychological Review, 73, 16-43.

Wu, M.-F., Suzuki, S. S., \& Siegel, J. M. (1988). Anatomical distribution and response patterns of reticular neurons active in relation to acoustic startle. Brain Research, 457, 399-406.

Yeomans, J. S., \& Frankl.and, P. W. (1996). The acoustic startle reflex: Neurons and connections. Brain Research Reviews, 21, 301-314.

(Manuscript received August 6, 1998; revision accepted for publication January 13, 1999.) 